\title{
The Compact, 1 kpc Host Galaxy of a Quasar at a Redshift of 7.1
}

\author{
Bram P. Venemans ${ }^{1}$, Fabian Walter ${ }^{1,2,3}$, Roberto Decarli ${ }^{1}$, Eduardo Bañados ${ }^{4,12}$, Jacqueline Hodge ${ }^{5}$, Paul Hewett ${ }^{6}$, \\ Richard G. McMahon ${ }^{6,7}$, Daniel J. Mortlock ${ }^{8,9,10}$, and Chris Simpson ${ }^{11}$ \\ ${ }^{1}$ Max-Planck Institute for Astronomy, Königstuhl 17, D-69117 Heidelberg, Germany; venemans@mpia.de \\ ${ }^{2}$ Astronomy Department, California Institute of Technology, MC105-24, Pasadena, CA 91125, USA \\ ${ }^{3}$ National Radio Astronomy Observatory, Pete V. Domenici Array Science Center, P.O. Box O, Socorro, NM 87801, USA \\ ${ }^{4}$ The Observatories of the Carnegie Institute of Washington, 813 Santa Barbara Street, Pasadena, CA 91101, USA \\ ${ }_{6}^{5}$ Leiden Observatory, Leiden University, P.O. Box 9513, NL2300 RA Leiden, The Netherlands \\ ${ }^{6}$ Institute of Astronomy, University of Cambridge, Madingley Road, Cambridge CB3 OHA, UK \\ ${ }^{7}$ Kavli Institute for Cosmology, University of Cambridge, Madingley Road, Cambridge CB3 OHA, UK \\ ${ }^{8}$ Astrophysics Group, Blackett Laboratory, Imperial College London, London, SW7 2AZ, UK \\ 9 Department of Mathematics, Imperial College London, London, SW7 2AZ, UK \\ ${ }^{10}$ Department of Astronomy, Stockholm University, Albanova, SE-10691 Stockholm, Sweden \\ ${ }^{11}$ Gemini Observatory, 670 N. A'ohoku Place, Hilo, HI 96720, USA \\ Received 2016 December 16; revised 2017 February 10; accepted 2017 February 12; published 2017 March 15
}

\begin{abstract}
We present Atacama Large Millimeter/submillimeter Array (ALMA) observations of the [C II] fine-structure line and the underlying far-infrared (FIR) dust continuum emission in J1120+0641, the most distant quasar currently known $(z=7.1)$. We also present observations targeting the $\mathrm{CO}(2-1), \mathrm{CO}(7-6)$, and [C I] $369 \mu \mathrm{m}$ lines in the same source obtained at the Very Large Array and Plateau de Bure Interferometer. We find a [C II] line flux of $F_{[\mathrm{C} \text { II] }}=1.11 \pm 0.10 \mathrm{Jy} \mathrm{km} \mathrm{s}^{-1}$ and a continuum flux density of $S_{227 \mathrm{GHz}}=0.53 \pm 0.04 \mathrm{mJy}_{\text {beam }}{ }^{-1}$, consistent with previous unresolved measurements. No other source is detected in continuum or [C II] emission in the field covered by ALMA $\left(\sim 25^{\prime \prime}\right)$. At the resolution of our ALMA observations $(0$ ". 23 , or $1.2 \mathrm{kpc}$, a factor of $\sim 70 \mathrm{smaller}$ beam area compared to previous measurements), we find that the majority of the emission is very compact: a high fraction $(\sim 80 \%)$ of the total line and continuum flux is associated with a region $1-1.5 \mathrm{kpc}$ in diameter. The remaining $\sim 20 \%$ of the emission is distributed over a larger area with radius $\lesssim 4 \mathrm{kpc}$. The [C II] emission does not exhibit ordered motion on kiloparsec scales: applying the virial theorem yields an upper limit on the dynamical mass of the host galaxy of $(4.3 \pm 0.9) \times 10^{10} M_{\odot}$, only $\sim 20 \times$ higher than the central black hole $(\mathrm{BH})$. The other targeted lines $(\mathrm{CO}(2-1), \mathrm{CO}(7-6)$, and $[\mathrm{C} \mathrm{I}])$ are not detected, but the limits of the line ratios with respect to the [C II] emission imply that the heating in the quasar host is dominated by star formation, and not by the accreting BH. The star formation rate (SFR) implied by the FIR continuum is $105-340 M_{\odot} \mathrm{yr}^{-1}$, with a resulting SFR surface density of $\sim 100-350 M_{\odot} \mathrm{yr}^{-1} \mathrm{kpc}^{-2}$, well below the value for Eddington-accretion-limited star formation.
\end{abstract}

Key words: cosmology: observations - galaxies: active - galaxies: high-redshift - galaxies: individual (ULAS J112001.48+064124.3) - galaxies: ISM

\section{Introduction}

Luminous quasars are powered by accreting, supermassive black holes (BHs). Quasars and thus supermassive BHs have been found at high redshifts, $z \sim 7$, when the universe was less than a billion years old (e.g., Mortlock et al. 2011; Venemans et al. 2013). These early, supermassive BHs are generally hosted by massive galaxies that form stars at a high rate. Indeed, locally there is a relation between the mass of the bulge and the mass of the $\mathrm{BH}$ in its center (see, e.g., Kormendy \& Ho 2013, for a review). An important question in astronomy is how the first $\mathrm{BHs}$ formed and whether the $\mathrm{BH}$ and hosting galaxy coevolved.

Over the past $15 \mathrm{yr}$, numerous surveys have established a sample of $\sim 100$ quasars at $z>6$ (e.g., Fan et al. 2006; Jiang et al. 2009, 2015; Mortlock et al. 2009; Willott et al. 2010b; Venemans et al. 2013, 2015; Carnall et al. 2015; Reed et al. 2015; Bañados et al. 2016; Matsuoka et al. 2016). The most luminous of these have BHs with masses in excess of $10^{9} M_{\odot}$ (e.g., Jiang et al. 2007; Kurk et al. 2007; Willott et al. 2010a; De Rosa et al. 2011, 2014; Venemans et al. 2015; Wu et al. 2015). As the accreting BH generally dominates the

\footnotetext{
${ }^{12}$ Carnegie-Princeton Fellow.
}

emission at rest-frame UV and optical wavelengths, observations at (sub)millimeter wavelength are needed to study the galaxies hosting these BHs. Several groups have targeted and detected $z \gtrsim 6$ quasars with millimeter facilities such as the IRAM Plateau de Bure Interferometer (PdBI) and Atacama Large Millimeter/submillimeter Array (ALMA) (e.g., Bertoldi et al. 2003; Maiolino et al. 2005; Wang et al. 2008, 2013; Willott et al. 2013, 2015; Venemans et al. 2016). These data show that rapid $\mathrm{BH}$ growth is, in some cases, accompanied by very high star formation rates (SFRs) of up to $\sim 1000 M_{\odot} \mathrm{yr}^{-1}$.

In this paper we investigate the host galaxy of the most distant quasar currently known, ULAS J112001.48+064124.3 (hereafter J1120+0641), at a redshift of $z=7.085$ (Mortlock et al. 2011). The quasar is powered by a $\mathrm{BH}$ with a mass of $(2.4 \pm 0.2) \times 10^{9} M_{\odot} \quad$ (Mortlock et al. 2011; De Rosa et al. 2014) and is accreting close to the Eddington limit (Mortlock et al. 2011; De Rosa et al. 2014; Barnett et al. 2015). The quasar host galaxy has previously been detected with the IRAM PdBI in [C II] and the dust continuum (Venemans et al. 2012). In these data the host galaxy was unresolved in a $\sim 2^{\prime \prime}$ beam and the dynamical mass and the morphology of the lineemitting gas could not be constrained. Here we present high spatial resolution $(0$ !" 23 , or $1.2 \mathrm{kpc})$ observations with ALMA 
(Section 2.1), decreasing the beam area by a factor of $\sim 70$. We also present observations with the PdBI (Section 2.2) and the NRAO Karl G. Jansky Very Large Array (VLA; Section 2.3) targeting the neutral and molecular gas lines $\mathrm{CO}(7-6)$, [C I] $369 \mu \mathrm{m}$, and $\mathrm{CO}(2-1)$. Our results are detailed in Section 3, followed by a discussion about the implications of our findings in Section 4. A summary is presented in Section 5.

Throughout this paper, we adopt a concordance cosmology with $H_{0}=70 \mathrm{~km} \mathrm{~s}^{-1} \mathrm{Mpc}^{-1}, \Omega_{M}=0.3$, and $\Omega_{\lambda}=0.7$, leading to a spatial scale of 5.2 proper kpc per arcsec at $z=7.085$. SFRs are calculated assuming a Kroupa (2001) initial mass function.

\section{Observations}

\subsection{ALMA Cycle 1 Observations}

The host galaxy of J1120+0641 was observed with ALMA between 2014 and 2015 June (program 2012.1.00882.S). In 2014 the antennas were in a compact configuration (33-36 antennas with baselines between 20 and $650 \mathrm{~m}$ ), and in 2015 they were in a more extended configuration, with baselines between 34 and $1574 \mathrm{~m}$ and a total of 38-47 antennas. J1120 +0641 was observed on 2014 June 9 for 33.5 minutes (on source), on 2014 June 10 for 26.8 minutes, and on 2014 June 11 for 33.5 minutes. The combined 2014 data reached an rms noise of $0.39 \mathrm{mJy} \mathrm{beam}^{-1}$ in a $20 \mathrm{MHz}$ bin, and the beam size was 0 ". $62 \times 0$ ". 51 (natural weighting). On 2015 June 26 and 27 , the quasar was observed for 33.4 minutes (on source) during both days. The beam size of the combined 2015 data was 0 "! $25 \times 0$ ". 24 (using natural weighting), and the rms noise per $20 \mathrm{MHz}$ bin is $0.21 \mathrm{mJy}_{\text {beam }}{ }^{-1}$. The full data set reached a noise of $0.15 \mathrm{mJy} \mathrm{beam}^{-1}(20 \mathrm{MHz})^{-1}$ and has a beam size of 0 " $31 \times 0$ ". 29 using natural weighting. Using a weighting factor of robust $=0.5$ results in a slightly higher rms noise of $0.17 \mathrm{mJy}^{-1}$ peam ${ }^{-1}$ per $20 \mathrm{MHz}$ bin and a beam size of 0 ! $23 \times 0$ "! 22 . This corresponds to $1.2 \mathrm{kpc}$ at the redshift of the quasar.

The ALMA observations covered the redshifted [C II] line at $\nu_{\text {obs }}=235.07 \mathrm{GHz}$ with two overlapping bandpasses of $1.875 \mathrm{GHz}$ each. The overlap was $20 \%$, which resulted in frequency coverage of $3.375 \mathrm{GHz}$ around the [C II] line. Two additional bandpasses of $1.875 \mathrm{GHz}$ each were placed around an observed frequency of $220 \mathrm{GHz}$. The data were reduced using Common Astronomy Software Applications (CASA; McMullin et al. 2007), following standard reduction steps.

\subsection{PdBI $3 \mathrm{~mm}$ Observations}

The $\operatorname{CO}(7-6) \quad\left(\nu_{\text {rest }}=806.652 \mathrm{GHz}\right)$ and $[\mathrm{C} \mathrm{I}]\left(\nu_{\text {rest }}=\right.$ $809.344 \mathrm{GHz}$ ) emission lines from $\mathrm{J} 1120+0641$ (redshifted to observed frequency around $\nu_{\mathrm{obs}} \sim 100 \mathrm{GHz}$ ) were targeted by the PdBI between 2011 July 4 and 2012 May 3. The observations were carried out with five to six antennas. The antennas were in the most compact $(D)$ configuration, providing a beam with a size of 4 !. $7 \times 4$ !' 2 . The WideX correlator that was used provided a continuous frequency coverage of $3.6 \mathrm{GHz}$ and covered both $\mathrm{CO}(7-6)$ and $[\mathrm{CI}]$ emission lines in a single setup. The data were reduced using the Grenoble Image and Line Data Analysis System software package. The total time on source was $18.3 \mathrm{hr}$ (six-antenna equivalent), resulting in an rms noise of $0.33 \mathrm{mJy}^{\text {beam }}{ }^{-1}$ per $20 \mathrm{MHz}$ bin. The continuum rms noise, measured in an image that was created by averaging all channels that do not cover the emission lines (resulting in a continuum bandwidth of $2.7 \mathrm{GHz}$; see Section 3.3), is $29 \mu \mathrm{Jy}$.

\subsection{VLA Observations}

We observed $\mathrm{CO}(2-1)$ in $\mathrm{J} 1120+0641$ (rest frequency $\nu_{\text {rest }}=230.5424 \mathrm{GHz}$, redshifted to $\left.\nu_{\mathrm{obs}}=28.5145 \mathrm{GHz}\right)$ as part of VLA project 11A-285. The observations were taken on 2013 January 12 and 13 in the DnC configuration (consisting of 27 antennas). We utilized the Ka-band receivers with the $\mathrm{A} / \mathrm{C}$ IF pair tuned to $32.008 \mathrm{GHz}$ and the $\mathrm{B} / \mathrm{D}$ IF pair tuned to $27.810 \mathrm{GHz}$. The observations were taken in full polarization mode with $16128 \mathrm{MHz}$ spectral windows (eight spectral windows per IF) and $642 \mathrm{MHz}$ channels per spectral window.

The observations consisted of a single pointing centered on the quasar host galaxy. We used fast-switching phase calibration (Carilli \& Holdaway 1999) on VLA calibrator $\mathrm{J} 1058+0133$ with a 4-minute cycle time, and the same source served as our bandpass calibrator. The bright source 3C 286 served as the flux calibrator for all observations. The observing time, including overhead, was $10 \mathrm{hr}$. The total time on source was $6.3 \mathrm{hr}$.

The data were reduced using the CASA package. We imaged the calibrated data using the clean algorithm with a cell size of $0 . " 5$ and natural weighting, resulting in a synthesized beam of 2 ". $3 \times 1$ "' 3 . The final cube has an $\mathrm{rms}$ of $15 \mu \mathrm{Jy}$ beam $^{-1}$ in a $76 \mathrm{MHz}$ window $\left(800 \mathrm{~km} \mathrm{~s}^{-1}\right)$ centered on the frequency of the expected CO line. The continuum rms is $4 \mu \mathrm{Jy}_{\text {beam }^{-1}}$.

\section{Results \\ 3.1. FIR Continuum}

In Figure 1 we show a $227 \mathrm{GHz}$ (observed wavelength $\lambda_{\text {obs }}=1.3 \mathrm{~mm}$ ) continuum map of the field that was created by averaging all channels at least $0.75 \mathrm{GHz}$ away from the expected [C II] emission line. As a consequence, the reconstructed continuum emission is dominated by ALMA's lowersideband observations (at frequencies $\sim 10 \mathrm{GHz}$ below the redshifted [C II] line). In the whole field (with a half-power beam width of 24 ". 8 ) only the quasar is detected (at signal-to-

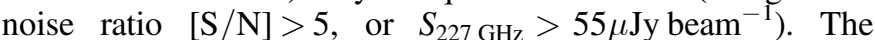
position (R.A. $=11^{\mathrm{h}} 20^{\mathrm{m}} 01^{\mathrm{s}} .465$; decl. $=+06^{\circ} 41^{\prime} 23^{\prime \prime}$. 810 ) is approximately $0 . / 5$ to the south of the position from the UK Infrared Telescope (UKIRT) Infrared Deep Sky Survey (UKIDSS) published in Mortlock et al. (2011). This difference could be due to a systematic difference in the absolute astrometric calibration between UKIDSS and ALMA. It is not possible to verify this, as there are no other sources in the field within the ALMA field-of-view beam, but similar offsets between ALMA and optical/near-infrared images have been reported (e.g., in the Hubble Ultra Deep Field; see Aravena et al. 2016; Rujopakarn et al. 2016). No offset from the UKIDSS position was seen in the PdBI data (Venemans et al. 2012). Within the uncertainties of the PdBI observations, the earlier millimeter continuum position was identical to that of the UKIDSS position.

The peak flux density of the quasar host is $S_{227 \mathrm{GHz}}=$ $0.26 \pm 0.01 \mathrm{mJy}$ beam $^{-1}$. To measure the total continuum flux density, we tapered the emission to 1 !' 9 . This resolution is similar to the beam of the original [C II] observations $(2$ !' $0 \times 1$ !! 7 ; Venemans et al. 2012). In this map, the peak flux density of the host is $S_{227 \mathrm{GHz}}=0.53 \pm 0.04 \mathrm{mJy}$ beam $^{-1}$. A similar value was 

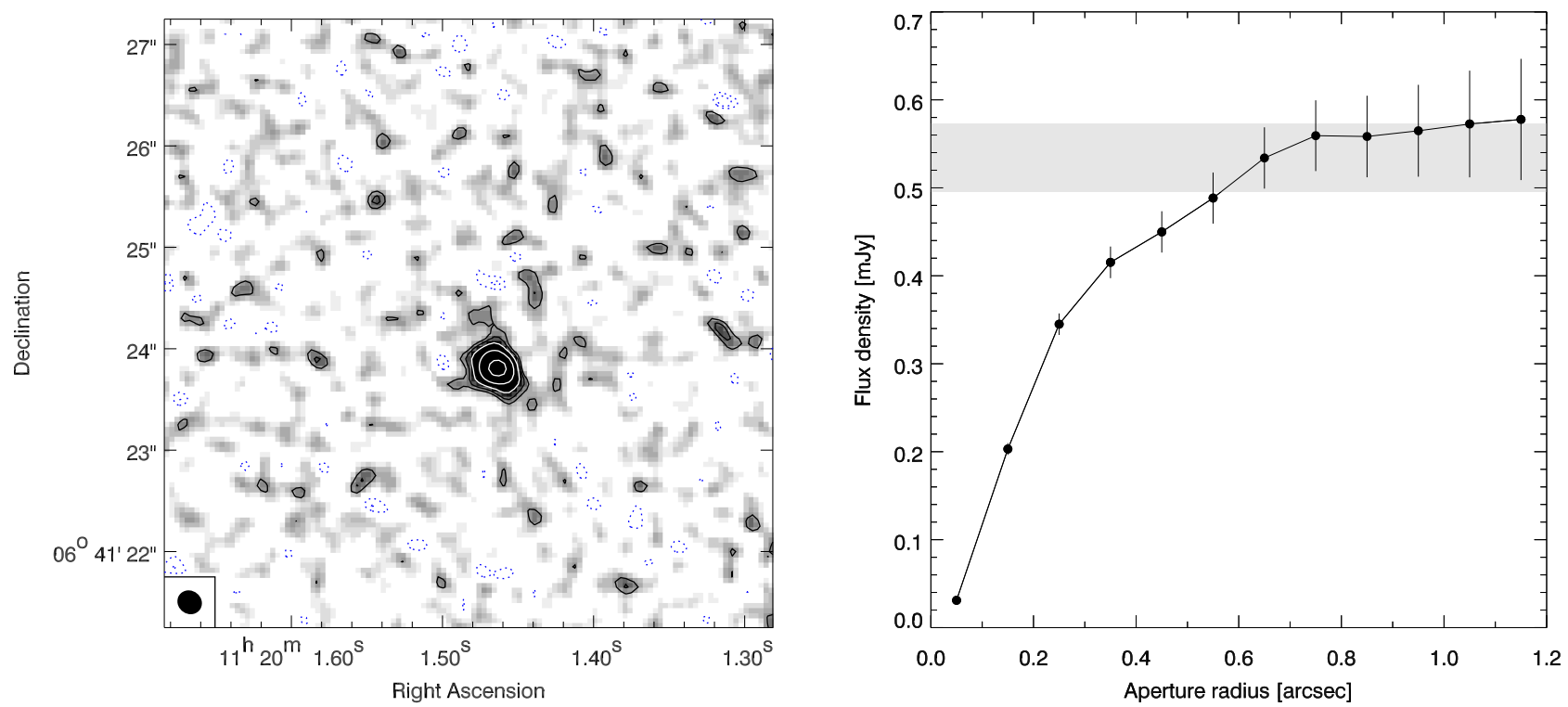

Figure 1. Left: $227 \mathrm{GHz}\left(\lambda_{\mathrm{obs}}=1.3 \mathrm{~mm}\right)$ continuum map of the field of $\mathrm{J} 1120+0641$. To create the map, all channels at least $0.75 \mathrm{GHz}$ away from the position of the [C II] emission line ( $\nu_{[\mathrm{C} \mathrm{II}], \mathrm{obs}} \approx 235 \mathrm{GHz}$; see Figure 2$)$ were averaged. The beam of $0.225 \times 0$. " 23 is shown in the bottom left corner. The $1 \sigma \mathrm{rms}$ noise in this map is $11 \mu \mathrm{Jy}$ beam $^{-1}$. The blue dashed contours are $-3 \sigma$ and $-2 \sigma$; the black solid contours are $+2 \sigma$ and $+3 \sigma$; the white solid contours are $+5 \sigma,+10 \sigma$, and $+20 \sigma$. Only the central quasar is detected $(\mathrm{S} / \mathrm{N} \sim 26)$. Right: estimated flux density of the central continuum source as a function of aperture radius. The flux density reaches a roughly constant value for apertures with radius $>0 . ! 7$, and the value is consistent within the errors with the peak flux density when observed with a beam of 1.9 (the gray band).

derived when performing aperture photometry (see Figure 1). For apertures larger than $\sim 0$ !' 7 the flux density is roughly constant and we measure a total flux density of $S_{227 \mathrm{GHz}}=0.56 \pm 0.04$ mJy (see Section 3.4). Note that due to the shape of the farinfrared (FIR) continuum, the continuum flux density is higher around the [C II] line (Figure 2; see also, e.g., Section 4.2 and Venemans et al. 2016). From the spectrum of the [C II] line (around an observed frequency of $235 \mathrm{GHz}$; Figure 2) we measure $S_{235 \mathrm{GHz}}=0.64 \pm 0.08 \mathrm{mJy}$ beam $^{-1}$, which is consistent with the published value of $S_{235 \mathrm{GHz}}=0.61 \pm 0.16 \mathrm{mJy}$ beam $^{-1}$ (Venemans et al. 2012).

We also created a map of the $100 \mathrm{GHz}\left(\lambda_{\text {obs }} \approx 3 \mathrm{~mm}\right)$ continuum emission from the PdBI data, using the channels that are expected to be line-free. At the position of the $227 \mathrm{GHz}$ continuum source we obtain a tentative $3 \sigma$ detection (flux density of $\left.S_{100 \mathrm{GHz}}=86 \pm 29 \mu \mathrm{Jy}\right)$. Based on the $1 \mathrm{~mm}$ continuum detection in the ALMA data, assuming an intrinsic dust temperature of 30-50 K (see Sections 4.1 and 4.2), an emissivity index of $\beta=1.6$, and taking the cosmic microwave background (CMB) into account (see, e.g., da Cunha et al. 2013; Venemans et al. 2016, Section 4.1), the expected flux density at $100 \mathrm{GHz}$ is $42-55 \mu \mathrm{Jy}$, consistent with our low$\mathrm{S} / \mathrm{N}$ measurement. The nondetection of the continuum in the VLA data $\left(S_{30} \mathrm{GHz}<11 \mu \mathrm{Jy}\right.$, Table 1$)$ is consistent with J1120+0641 being radio-quiet (Momjian et al. 2014).

\section{2. [C II] Emission Line}

The [C II] emission line is detected at high $\mathrm{S} / \mathrm{N}$ in the ALMA data. To get an estimate of the total line flux, we tapered the data cube to a beam of 1.9 . We show the spectrum from the peak pixel in this cube, together with a Gaussian fit, in Figure 2 (top). The redshift of the [C II] line is $z_{[\mathrm{C} \mathrm{II}}=7.0851 \pm 0.0005$, the peak flux density is $f_{\mathrm{p}}=2.60 \pm 0.25 \mathrm{mJy} \mathrm{beam}^{-1}$, and the FWHM is $\mathrm{FWHM}_{[\mathrm{C} \mathrm{II}}=400 \pm 45 \mathrm{~km} \mathrm{~s}^{-1}$ (see also Table 1). The line flux of $F_{[\mathrm{CI}]}=1.11 \pm 0.10 \mathrm{Jy} \mathrm{km} \mathrm{s}^{-1}$ is consistent

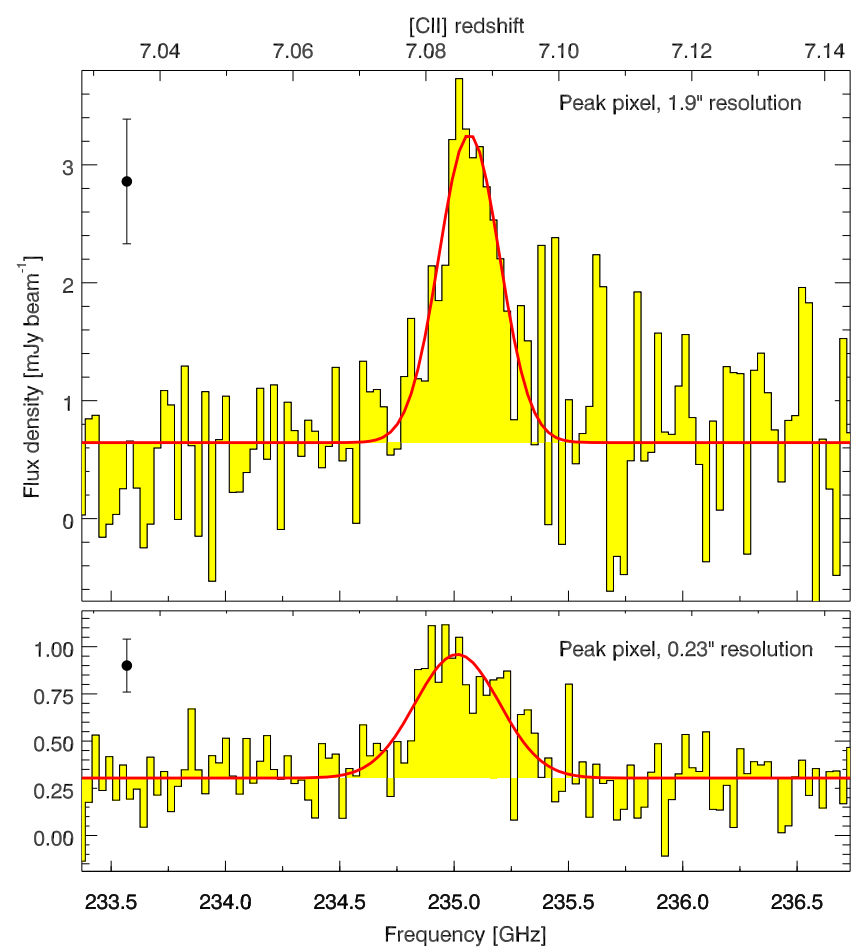

Figure 2. Top: spectrum extracted from the peak pixel in the ALMA data cube tapered to a spatial resolution of 1 !" 9 . The bins are $30 \mathrm{MHz}$, which corresponds to $\sim 38 \mathrm{~km} \mathrm{~s}^{-1}$. The typical $1 \sigma$ uncertainty per bin of $0.52 \mathrm{mJy} \mathrm{beam}^{-1}$ is plotted in the top left corner. The red line is a Gaussian+constant fit to the spectrum. Bottom: same as above, but this spectrum is extracted from the peak pixel in the data cube with the full spatial resolution $(0$ !' 23). The rms noise is $0.14 \mathrm{mJy}$ beam $^{-1}$ per $30 \mathrm{MHz}$ bin.

with the value of $F_{[\mathrm{C} \mathrm{II}]}=1.03 \pm 0.14 \mathrm{Jy} \mathrm{km} \mathrm{s}^{-1}$ published by Venemans et al. (2012), while the line width measured in the ALMA data is a factor of $1.69 \pm 0.32$ larger than the earlier value that was based on lower-S/N data. The [C II] rest-frame 
Table 1

Observed Properties of $\mathrm{J} 1120+0641$

\begin{tabular}{|c|c|}
\hline R.A. (J2000) & $11^{\mathrm{h}} 20^{\mathrm{m}} 01^{\mathrm{s}} .465$ \\
\hline Decl. (J2000) & $+06^{\circ} 41^{\prime} 23^{\prime \prime} 810$ \\
\hline$z_{[\text {[ III }]}$ & $7.0851 \pm 0.0005$ \\
\hline$F_{[\mathrm{C} \mathrm{II}]}\left(\mathrm{Jy} \mathrm{km} \mathrm{s}^{-1}\right)$ & $1.11 \pm 0.10$ \\
\hline $\mathrm{FWHM}_{[\mathrm{C} \mathrm{II}]}\left(\mathrm{km} \mathrm{s}^{-1}\right)$ & $400 \pm 45$ \\
\hline$S_{227 \mathrm{GHz}}(\mathrm{mJy})$ & $0.53 \pm 0.04$ \\
\hline$S_{100 \mathrm{GHz}}(\mathrm{mJy})$ & $0.086 \pm 0.029$ \\
\hline$S_{30 \mathrm{GHz}}(\mathrm{mJy})$ & $<0.011$ \\
\hline $\mathrm{EW}_{[\mathrm{C} \text { II] }}(\mu \mathrm{m})$ & $0.90 \pm 0.14$ \\
\hline$F_{\mathrm{CO}(2-1)}\left(\mathrm{Jy} \mathrm{km} \mathrm{s}^{-1}\right)$ & $<0.034$ \\
\hline$F_{\mathrm{CO}(7-6)}\left(\mathrm{Jy} \mathrm{km} \mathrm{s}^{-1}\right)$ & $<0.20$ \\
\hline$F_{[\mathrm{C} \text { I] }}\left(\mathrm{Jy} \mathrm{km} \mathrm{s}^{-1}\right)$ & $<0.20$ \\
\hline Size continuum ${ }^{\mathrm{a}}\left(\operatorname{arcsec}^{2}\right)$ & $(0.23 \pm 0.03) \times(0.16 \pm 0.03)$ \\
\hline Size continuum ${ }^{\mathrm{a}}\left(\mathrm{kpc}^{2}\right)$ & $(1.24 \pm 0.14) \times(0.83 \pm 0.14)$ \\
\hline Size $[\mathrm{C}$ II $]$ emission $^{\mathrm{a}}\left(\operatorname{arcsec}^{2}\right)$ & $(0.31 \pm 0.05) \times(0.27 \pm 0.05)$ \\
\hline Size $[\mathrm{C}$ II $]$ emission $^{\mathrm{a}}\left(\mathrm{kpc}^{2}\right)$ & $(1.65 \pm 0.29) \times(1.44 \pm 0.26)$ \\
\hline
\end{tabular}

Note.

${ }^{\mathrm{a}}$ The sizes listed here are diameters and are derived for the central component that contains around $80 \%$ of the total flux density (see Section 3.4).

equivalent width is $\left.\mathrm{EW}_{[\mathrm{C}} \mathrm{II}\right]=0.90 \pm 0.14 \mu \mathrm{m}$. This is only $\sim 30 \%$ lower than that of local starburst galaxies (e.g., DíazSantos et al. 2013; Sargsyan et al. 2014).

Also shown in Figure 2 (bottom) is the spectrum of the peak pixel in the high-resolution data cube (with a beam of 0 !' $23 \times 0$ ". 22). The [C II] line is significantly fainter in the center, with a peak flux density $f_{\mathrm{p}}=0.66 \pm 0.06 \mathrm{mJy} \mathrm{beam}^{-1}$ and a flux of $F_{[\mathrm{C} \text { II] }}=0.39 \pm 0.04 \mathrm{Jy} \mathrm{km} \mathrm{s}^{-1}$, which is $35 \%$ of the total line flux. This means that the [C II] line is spatially resolved in our data (see Section 3.4). The peak of the [C II] emission coincides with the peak of the continuum emission (Figure 3). The line width is broader in the center, with an $\mathrm{FWHM}_{[\mathrm{C} \text { II] }}=555 \pm 60 \mathrm{~km} \mathrm{~s}^{-1}$. We will come back to this in Section 4.4 .

\subsection{Limits on $\mathrm{CO}$ and [CI] Emission}

We searched for $\mathrm{CO}(7-6)$ and $[\mathrm{CI}]$ emission in the PdBI $3 \mathrm{~mm}$ data. After creating a continuum-subtracted data cube using the CASA task "uvcontsub," we averaged the channels where we expected the $\mathrm{CO}(7-6)$ and $[\mathrm{CI}$ lines, based on the [C II] redshift, over $400 \mathrm{~km} \mathrm{~s}^{-1}$ (the [C II] line FWHM; see Table 1). In the resulting maps (not shown here) no significant $(>3 \sigma)$ line emission was detected at the location of the quasar host galaxy. For the $\mathrm{CO}(7-6)$ and $[\mathrm{CI}$ lines we derived $3 \sigma$ upper limits on the line strength of $\mathrm{F}_{\mathrm{CO}(7-6),[\mathrm{CI}]}<$ $0.20 \mathrm{Jy} \mathrm{km} \mathrm{s}^{-1}$.

Similarly, we derived an upper limit on the $\mathrm{CO}(2-1)$ line from the VLA data. Averaging the data over $400 \mathrm{~km} \mathrm{~s}^{-1}$ resulted in a map with no significant emission at the position of the quasar host. The rms of the image was $0.021 \mathrm{mJy}$, and the $3 \sigma$ upper limit on the $\mathrm{CO}(2-1)$ line was $F_{\mathrm{CO}(2-1)}<$ $0.034 \mathrm{Jy} \mathrm{km} \mathrm{s}^{-1}$. We will discuss the implications of these nondetections in Section 4.1.

\footnotetext{
${ }^{13}$ These upper limits are corrected to account for flux in the outer line wings that are not included when averaging over the FWHM of a Gaussian line.
}

\subsection{Size and Structure of the Emission Regions}

Continuum: to estimate the size of the continuum-emitting region, we fitted a 2D Gaussian to the continuum map using the CASA task "imfit." The quasar host galaxy is marginally resolved, and we derive a deconvolved size with an FWHM of $(0.23 \pm 0.03) \times(0.16 \pm 0.03) \operatorname{arcsec}^{2}$, or $(1.24 \pm 0.14) \times$ $(0.83 \pm 0.14) \mathrm{kpc}^{2}$. The integrated flux density of this central component is $0.43 \pm 0.03 \mathrm{mJy}$, which is $\sim 80 \%$ of the peak measured in the tapered continuum image (Section 3.1). The remaining $20 \%$ of the total continuum flux density comes from a more extended region, with a size $\lesssim 0$." 6 in radius $(\lesssim 3 \mathrm{kpc}$; see Figure 1).

$[C \mathrm{II}]$ line: in order to measure the size of the [C II]-emitting region, we first created a continuum-subtracted data cube: we fitted a first-order polynomial to the channels at least $0.75 \mathrm{GHz}$ away from the line center and subtracted this continuum from the data using the CASA task "uvcontsub." A map of the [C II] emission was produced by averaging the cube over $600 \mathrm{~km} \mathrm{~s}^{-1}\left(1.5 \times \mathrm{FWHM}_{[\mathrm{CI}}\right)$ around the peak of the [C II] emission. The result is shown in Figure 3. From a 2D Gaussian fit to this map we obtained a deconvolved size of the $[\mathrm{C}$ II $]$-emitting region of $(0.31 \pm 0.05) \times(0.27 \pm 0.05)$ $\operatorname{arcsec}^{2}$ in diameter, which corresponds to $(1.65 \pm 0.29) \times$ $(1.44 \pm 0.26) \mathrm{kpc}^{2}$. The area of the resolved [C II]-emitting region of $1.9 \pm 0.5 \mathrm{kpc}^{2}$ is larger than the area of the continuum region $\left(0.8 \pm 0.2 \mathrm{kpc}^{2}\right)$. A larger extent of [C II] emission compared to that of the continuum emission has also been reported in other $z \gtrsim 6$ quasar host galaxies (e.g., Wang et al. 2013; Venemans et al. 2016). The total flux density of the resolved component is $1.35 \pm 0.15 \mathrm{mJy}$, which corresponds to a flux of $0.81 \pm 0.09 \mathrm{Jy} \mathrm{km} \mathrm{s}^{-1}$.

We also performed aperture photometry on the [C II] image. We recovered all the flux measured in the tapered spectrum within a radius of $0 . " 8(\sim 4.3 \mathrm{kpc})$. At larger aperture radii we tentatively detected additional flux. We estimated that up to $20 \%$ of additional line flux might be present at scales up to $\sim 7 \mathrm{kpc}$ from the quasar, although the significance is low $(1 \sigma)$.

Bright [C II] emission in $z \gtrsim 6$ quasar hosts often shows indications of rotation (e.g., Wang et al. 2013; Willott et al. 2013; Venemans et al. 2016). To investigate whether the gas in $\mathrm{J} 1120+0641$ displays ordered motion, we separately mapped the blue and red sides of the emission line; see Figure 3. The blue- and redshifted emission peaks coincide with the continuum emission. It therefore appears that the gas traced by [C II] emission does not show rotation on scales of $\gtrsim 1 \mathrm{kpc}$. We will discuss the implications of this in Section 4.4.

A summary of our results, described in Sections 3.1-3.4, is listed in Table 1.

\section{Discussion}

\subsection{Origin of the Heating Radiation}

We now compare our (limits on the) emission-line ratios to models to constrain the physical parameters of the emitting gas (see, e.g., Kaufman et al. 1999; Meijerink \& Spaans 2005; Meijerink et al. 2007). In particular, the line ratio $[\mathrm{CII}] /[\mathrm{CI}]$ can be used to determine the dominant source of radiation, the hard X-ray radiation of the accreting, supermassive $\mathrm{BH}$ (an X-ray-dominated region, or XDR), or UV radiation from hot stars (a photon-dominated region, or PDR) (see Figure 4). To calculate the intrinsic line ratios, we first need to determine the intrinsic luminosity of the emission lines. Because we are 

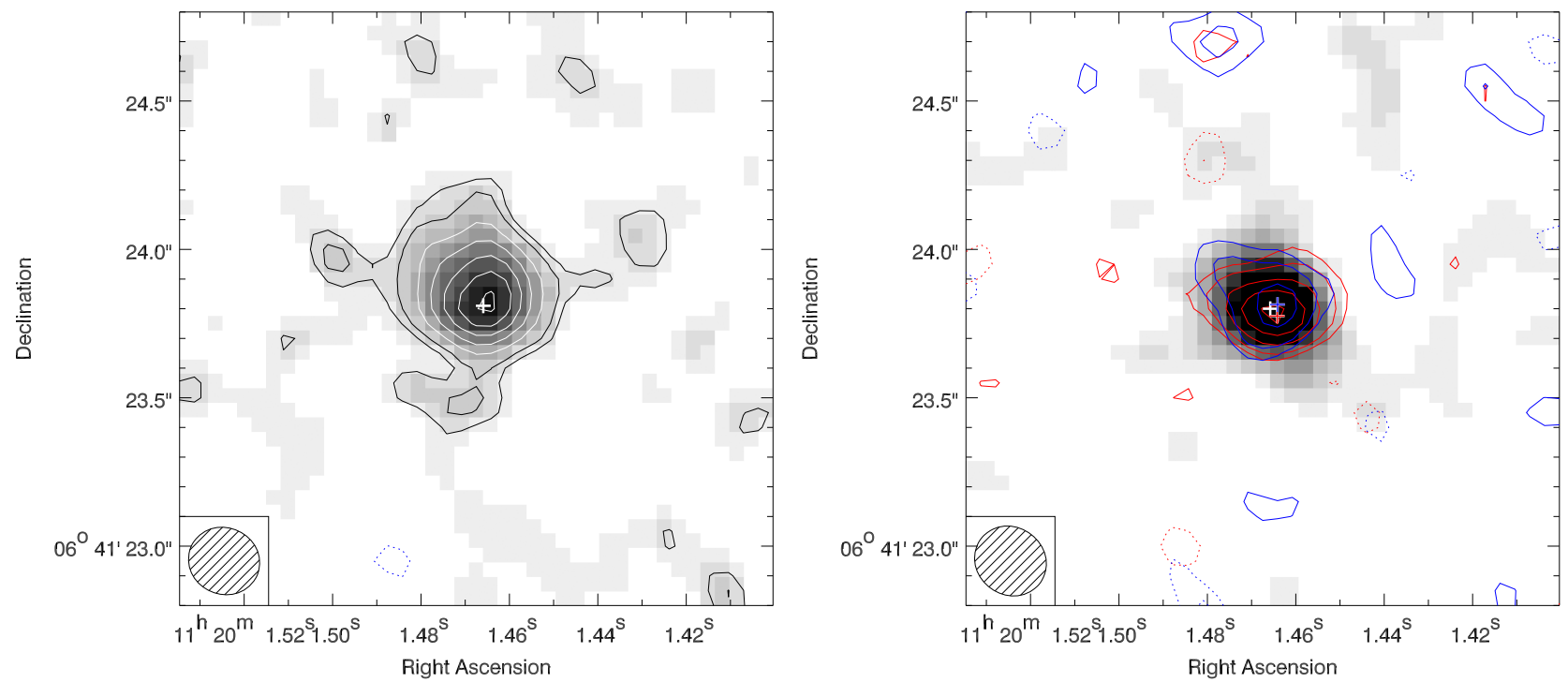

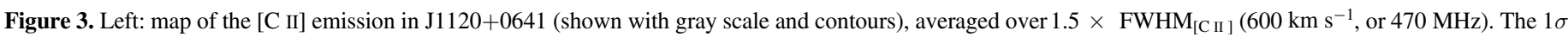

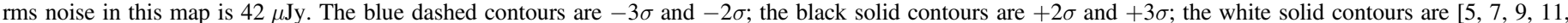

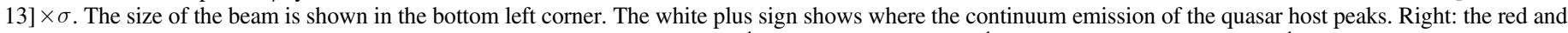

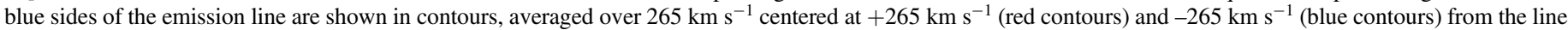

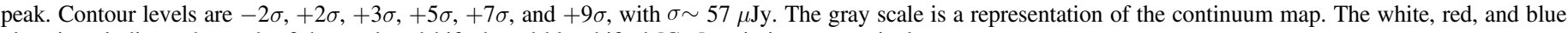
plus signs indicate the peak of the total, redshifted, and blueshifted [C II] emission, respectively.

measuring the flux of the lines against the CMB, the intrinsic luminosity will depend on the excitation temperature (da Cunha et al. 2013): $F_{\text {in }}=F_{\text {obs }} /\left\{1-B_{\nu}\left[T_{\mathrm{CMB}}(z=7.0851)\right] / B_{\nu}\left[T_{\mathrm{ex}}\right]\right\}$, where $F_{\text {obs }}$ and $F_{\text {in }}$ are the observed and intrinsic line flux, $B_{\nu}$ the Planck function at the rest-frame frequency $\nu$ of the line, $T_{\mathrm{CMB}}(z=7.0851)$ the temperature of the $\mathrm{CMB}$ at redshift $z=7.0851(\approx 22 \mathrm{~K})$, and $T_{\mathrm{ex}}$ the excitation temperature.

If collisions dominate the excitation, then the excitation temperature is set by the kinetic temperature of the gas. In this paper we further assume thermodynamic equilibrium between the dust and the gas, i.e., $T_{\text {gas }}=T_{\text {dust }}$. This assumption is motivated by the study of dust and $\mathrm{CO}$ emission in the host galaxy of quasar $\mathrm{J} 1148+5251$ at $z=6.42$ in which $T_{\mathrm{ex}} \approx T_{\text {dust }}$ (Beelen et al. 2006; Riechers et al. 2009; Stefan et al. 2015). We also assume that the dust has a constant temperature throughout the host galaxy. To explore the range of luminosities and line ratios in $\mathrm{J} 1120+0641$, we derive intrinsic luminosities of the emission lines for various temperatures that are found in the literature (Table 2). Several studies of $z \sim 6$ quasar host galaxies (e.g., Wang et al. 2013; Willott et al. 2013, 2015 ) implement a dust temperature of $T_{\mathrm{d}}=47 \mathrm{~K}$ as derived by Beelen et al. (2006) for distant luminous quasars. A study by Priddey \& McMahon (2001) found an average dust temperature in quasars of $T_{\mathrm{d}}=41 \mathrm{~K}$. We also computed the line luminosities in the case of a lower temperature of $T_{\mathrm{d}}=30 \mathrm{~K}$ (Walter et al. 2011; Venemans et al. 2016). Alternatively, the gas temperature could be much higher than that of the dust, $T_{\text {gas }} \gg 100 \mathrm{~K}$ (e.g., Contursi et al. 2013), and the effect of the CMB becomes negligible (the "no CMB" column in Table 2).

We can compare the limits on the $[\mathrm{C} \mathrm{II}] /[\mathrm{C} \mathrm{I}]$ ratio in $\mathrm{J} 1120$ +0641 (Table 2) to those from PDR and XDR models (Figure 4). In PDR models, the [C II]/[C I] ratio covers a large range from $\sim 3$ to 50 , depending on the density and strength of the UV radiation field (Kaufman et al. 1999; Meijerink et al. 2007). On the other hand, in XDRs the $[\mathrm{C} \mathrm{II}] /[\mathrm{C} \mathrm{I}]$ ratio is generally lower than in a PDR, with a maximum of around $\sim 6$ (Meijerink et al. 2007). We measure a lower limit on the $[\mathrm{C} \mathrm{II}] /[\mathrm{C} \mathrm{I}]$ line ratio of $\sim 8.7$. Therefore, based on the XDR models, we can exclude that the radiation illuminating the gas is dominated by hard X-ray radiation from the accreting $\mathrm{BH}$, but instead should mainly come from hot stars.

\subsection{Infrared Luminosity and SFRs}

To compute the FIR luminosity, we have to assume a shape of the dust emission. The cold dust spectral energy distribution (SED) of distant quasar host galaxies is often parameterized as an optically thin modified blackbody (e.g., Priddey \& McMahon 2001; Beelen et al. 2006; Leipski et al. 2014) with a dust temperature $T_{\mathrm{d}}$ and emissivity index $\beta$. Adopting $T_{\mathrm{d}}=47 \mathrm{~K}$ and $\beta=1.6$ (Beelen et al. 2006), integrating the dust SED from 42.5 to $122.5 \mu \mathrm{m}$, and taking the CMB into account, we derive an FIR luminosity of $L_{\mathrm{FIR}}=$ $(1.5 \pm 0.1) \times 10^{12} L_{\odot}$. For $T_{\mathrm{d}}=41 \mathrm{~K}$ and $\beta=1.95$ (Priddey \& McMahon 2001), we derive a similar value for the FIR luminosity. A lower dust temperature of $30 \mathrm{~K}$ (Venemans et al. $2016)$ and $\beta=1.6$ results in $L_{\mathrm{FIR}}=(5.6 \pm 0.4) \times 10^{11} L_{\odot}$ (see Table 2 for a summary).

In Section 4.1 we concluded that the gas is predominantly heated by UV radiation from stars. If that also applies to the dust, then we can use the infrared luminosity to constrain the SFR of the host galaxy. This is supported by Barnett et al. (2015), who analyzed the full SED of J1120+0641 and concluded that the emission around $235 \mathrm{GHz}$ in the rest frame is dominated by a cool dust component and not by the accreting $\mathrm{BH}$.

To estimate the SFR from the continuum detection, we first integrated the modified blackbody from 8 to $1000 \mu \mathrm{m}$ to obtain the total infrared luminosity $L_{\mathrm{TIR}}$. Depending on the parameters of the modified blackbody, we derive total infrared luminosities ranging from $L_{\mathrm{FIR}}=(7.7 \pm 0.6) \times 10^{11} L_{\odot}$ to 

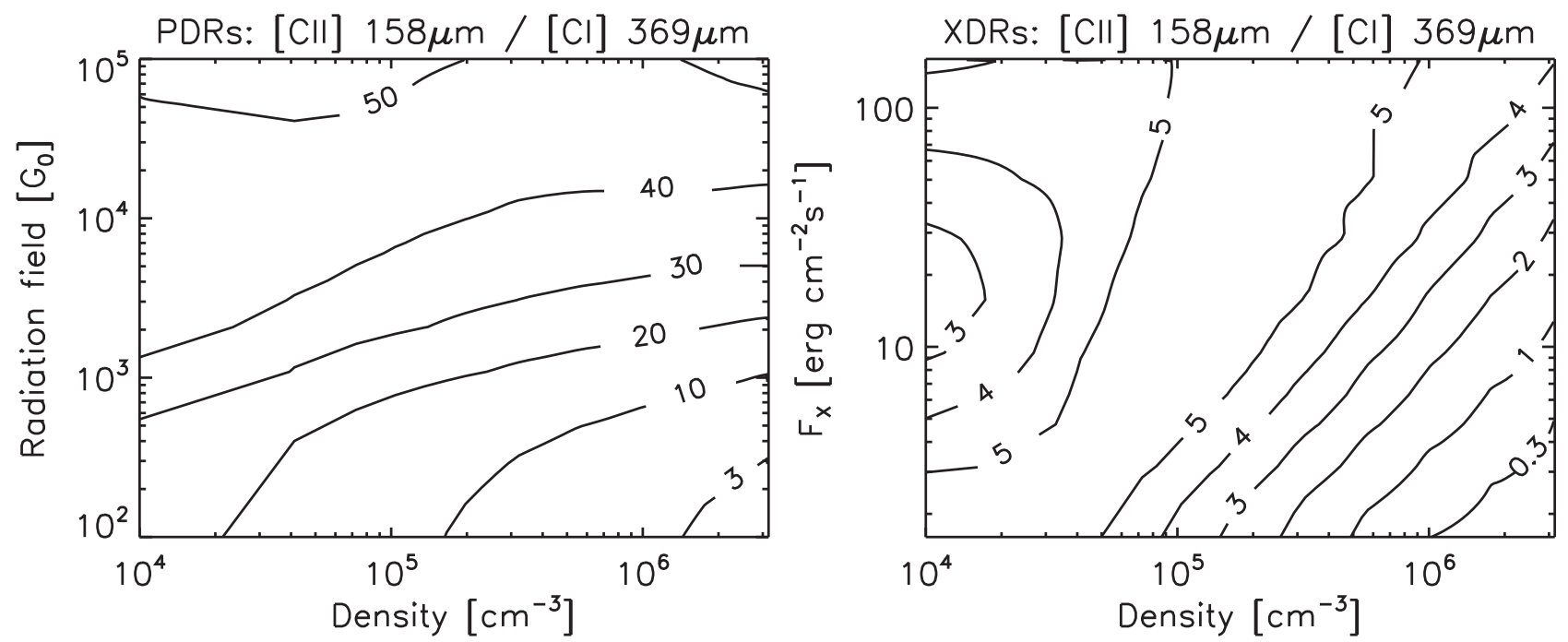

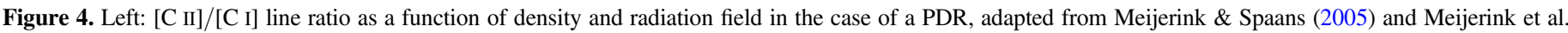

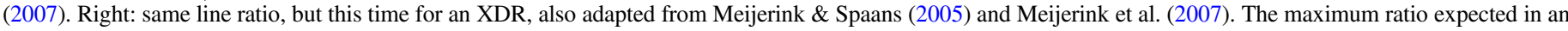

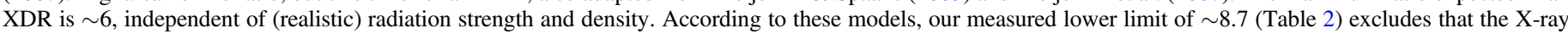
radiation of the quasar dominates the gas heating.

$L_{\mathrm{FIR}}=(2.1 \pm 0.2) \times 10^{12} L_{\odot}($ Table 2$)$. We then applied the local scaling relation between the total infrared luminosity and SFR from Murphy et al. (2011): $\mathrm{SFR}_{\mathrm{TIR}}=L_{\mathrm{TIR}} / 6.7 \times 10^{9}$ with $\mathrm{SFR}_{\mathrm{TIR}}$ in units of $M_{\odot} \mathrm{yr}^{-1}$ and $L_{\mathrm{TIR}}$ in units of $L_{\odot}$. We estimate an SFR of $105-340 M_{\odot} \mathrm{yr}^{-1}$ (Table 2), where the main uncertainty is the shape of the dust SED. In Section 3.4 we concluded that $\sim 80 \%$ of the continuum emission originates from a region that measures $1.2 \mathrm{kpc} \times 0.8 \mathrm{kpc}$ in diameter, or $0.8 \mathrm{kpc}^{2}$. This means that the star formation rate density (SFRD) is $\sim 100-350 M_{\odot} \mathrm{yr}^{-1} \mathrm{kpc}^{2}$. The lower limit is an order of magnitude smaller than the SFRD derived for the more FIRluminous bright quasar $\mathrm{J} 1148+5251$ at $z=6.42$ (Walter et al. 2009), in which the SFRD approaches the Eddington limit for star formation.

Alternatively, we can compute the SFR from the luminosity of the [C II] line. Applying the [C II] SFR conversion for highredshift sources from De Looze et al. (2014), we derive an $\mathrm{SFR}_{[\mathrm{C} \text { II] }}=70-700 \quad M_{\odot} \mathrm{yr}^{-1} \quad($ Table 2$)$. Within the large uncertainties, this value is consistent with the one derived from the FIR continuum emission.

\subsection{Dust and Gas Mass}

Following Venemans et al. (2012), we derived the dust mass from the FIR luminosity assuming a temperature and a dust mass opacity coefficient: $M_{\text {dust }} \sim S_{\nu} /\left[\kappa_{\lambda} \times B_{\nu}\left(T_{\mathrm{d}}\right)\right]$ (e.g., Hildebrand 1983) with $S_{\nu}$ the continuum flux density at rest-frame frequency $\nu$ and the dust mass opacity $\kappa_{\lambda}=$ $0.77(850 \mu \mathrm{m} / \lambda)^{\beta} \mathrm{cm}^{2} \mathrm{~g}^{-1}$ (Dunne et al. 2000). For the range of temperatures and emissivity indices considered in this paper, our best estimate for the dust mass in J1120+0641 is $(0.8-4) \times 10^{8} M_{\odot}($ Table 2$)$.

A limit on the molecular gas mass can be derived from the upper limit on the $\mathrm{CO}$ luminosity $L_{\mathrm{CO}(2-1)}^{\prime}$ (in units of $\mathrm{K} \mathrm{km} \mathrm{s}^{-1} \mathrm{pc}^{2}$ ) using $L_{\mathrm{CO}(1-0)}^{\prime} \approx L_{\mathrm{CO}(2-1)}^{\prime}$ (e.g., Carilli \& Walter 2013) and applying a conversion factor of $\alpha=M_{\mathrm{gas}} / L_{\mathrm{CO}(1-0)}^{\prime}=0.8$ $M_{\odot}\left(\mathrm{K} \mathrm{km} \mathrm{s}^{-1} \mathrm{pc}^{2}\right)^{-1}$ as found for nearby ULIRGs (e.g., Downes \& Solomon 1998). Taking the effects of the CMB into account, our VLA upper limit on the $\mathrm{CO}(2-1)$ line flux results in an upper limit on the CO luminosity of $L_{\mathrm{CO}(1-0)}^{\prime}<4 \times 10^{10} \mathrm{~K} \mathrm{~km} \mathrm{~s}^{-1} \mathrm{pc}^{2}$ (see Table 2) and a gas mass limit of $M_{\text {gas }}<3 \times 10^{10} M_{\odot}$. Alternatively, we can use the PdBI upper limit on the $\mathrm{CO}(7-6)$ line flux to derive $L_{\mathrm{CO}(1-0)}^{\prime}$. Applying a $\mathrm{CO}$ excitation ladder that is similar to that in quasar $\mathrm{J} 1146+5251$ at $z=6.42$ (e.g., Stefan et al. 2015), we derive an upper limit of $M_{\text {gas }} \lesssim 2 \times 10^{10} M_{\odot}$.

If we combine these upper limits with our estimates of the dust mass, we derive gas-to-dust mass ratio limits of $<80-250$ (strongly depending on the dust and gas temperature; see Table 2), which are consistent with locally derived values of 100 (e.g., Draine et al. 2007; Sandstrom et al. 2013).

\subsection{Dynamical Mass Estimate}

The dynamical mass of $z>6$ quasar hosts has often been computed by assuming that the gas is rotating in an inclined disk (e.g., Walter et al. 2003; Wang et al. 2013; Willott et al. 2015; Venemans et al. 2016). This approach was motivated by the detection of velocity gradients in the [C II]-emitting gas. From Figure 3 it is clear that in $\mathrm{J} 1120+0641$ there is no evidence for rotation on scales of $\gtrsim 1 \mathrm{kpc}$. Instead, we here use the virial theorem to estimate a dynamical mass of the quasar host galaxy: $M_{\text {dyn }}=3 R \sigma^{2} / 2 G$, with $R$ the radius of the lineemitting region, $\sigma$ the velocity dispersion of the gas, and $G$ the gravitational constant. In Section 3.4 we measure a maximum radius of $4.3 \mathrm{kpc}$, and from the tapered spectrum in Figure 2 we obtain a velocity dispersion of $\sigma=169 \pm 18 \mathrm{~km} \mathrm{~s}^{-1}$, which results in an upper limit on the dynamical mass of $M_{\text {dyn }}=(4.3 \pm 0.9) \times 10^{10} M_{\odot}$, which is similar to dynamical masses derived for other $z \gtrsim 6$ quasar host galaxies (e.g., Walter et al. 2009; Wang et al. 2013; Willott et al. 2015; Venemans et al. 2016). Intriguingly, the $\mathrm{BH}$ with a mass of ${ }^{14}$ $M_{\mathrm{BH}}=(2.4 \pm 0.2) \times 10^{9} M_{\odot}$ (De Rosa et al. 2014) already contains $\sim 6 \%$ of this dynamical mass. This fraction is about $10 \times$ higher than the mass ratio of $\mathrm{BHs}$ and bulges in local early-type galaxies $\left(M_{\mathrm{BH}} / M_{\text {bulge }}=0.49 \%\right.$; Kormendy \& Ho 2013). High BH-to-dynamical mass ratios are also found in other $z \gtrsim 6$ quasar host galaxies, which have BH-to-

\footnotetext{
14 The uncertainty quoted here does not include the systematic uncertainty of a factor of $\sim 3$ in the method applied by De Rosa et al. (2014) to derive the mass of $\mathrm{BH}$.
} 
Table 2

Derived Properties of the Host Galaxy of Quasar J1120+0641 at $z=7.0851$ as a Function of Temperature $T_{\mathrm{d}}$ and Emissivity Index $\boldsymbol{\beta}$

\begin{tabular}{|c|c|c|c|c|}
\hline Parameter & $T_{\mathrm{d}}=47 \mathrm{~K}, \beta=1.6$, no CMB & $T_{\mathrm{d}}=47 \mathrm{~K}, \beta=1.6$ & $T_{\mathrm{d}}=41 \mathrm{~K}, \beta=1.95$ & $T_{\mathrm{d}}=30 \mathrm{~K}, \beta=1.6$ \\
\hline$\overline{L_{\mathrm{FIR}}\left(L_{\odot}\right)}$ & $(1.3 \pm 0.1) \times 10^{12}$ & $(1.5 \pm 0.1) \times 10^{12}$ & $(1.5 \pm 0.1) \times 10^{12}$ & $(5.6 \pm 0.4) \times 10^{11}$ \\
\hline$L_{\mathrm{TIR}}\left(L_{\odot}\right)$ & $(1.9 \pm 0.1) \times 10^{12}$ & $(2.1 \pm 0.2) \times 10^{12}$ & $(1.9 \pm 0.1) \times 10^{12}$ & $(7.7 \pm 0.6) \times 10^{11}$ \\
\hline$M_{\text {dust }}\left(M_{\odot}\right)$ & $(8.6 \pm 0.6) \times 10^{7}$ & $(9.6 \pm 0.7) \times 10^{7}$ & $(7.7 \pm 0.6) \times 10^{7}$ & $(4.2 \pm 0.3) \times 10^{8}$ \\
\hline$L_{[\mathrm{C} \mathrm{II]}}\left(L_{\odot}\right)$ & $(1.3 \pm 0.1) \times 10^{9}$ & $(1.5 \pm 0.1) \times 10^{9}$ & $(1.6 \pm 0.1) \times 10^{9}$ & $(2.0 \pm 0.2) \times 10^{9}$ \\
\hline$L_{[\mathrm{C} \mathrm{I}]}\left(L_{\odot}\right)$ & $<1.0 \times 10^{8}$ & $<1.4 \times 10^{8}$ & $<1.5 \times 10^{8}$ & $<2.3 \times 10^{8}$ \\
\hline$L_{\mathrm{CO}(2-1)}\left(L_{\odot}\right)$ & $<5.0 \times 10^{6}$ & $<8.4 \times 10^{6}$ & $<9.4 \times 10^{6}$ & $<1.6 \times 10^{7}$ \\
\hline$L_{\mathrm{CO}(7-6)}\left(L_{\odot}\right)$ & $<1.0 \times 10^{8}$ & $<1.4 \times 10^{8}$ & $<1.5 \times 10^{8}$ & $<2.3 \times 10^{8}$ \\
\hline$L_{[\mathrm{C} \mathrm{II]}} / L_{[\mathrm{C} \mathrm{I}]}$ & $>13.0$ & $>10.6$ & $>10.1$ & $>8.7$ \\
\hline$L_{\mathrm{CO}(1-0)}^{\prime}{ }^{\mathrm{a}}\left(\mathrm{K} \mathrm{km} \mathrm{s}^{-1} \mathrm{pc}^{2}\right)$ & $<1.3 \times 10^{10}$ & $<2.1 \times 10^{10}$ & $<2.4 \times 10^{10}$ & $<4.0 \times 10^{10}$ \\
\hline$L_{[\mathrm{C} \mathrm{II]}} / L_{\mathrm{CO}(1-0)^{\mathrm{a}}}$ & $>2200$ & $>1400$ & $>1300$ & $>1000$ \\
\hline$M_{\mathrm{gas}} / M_{\mathrm{dust}}$ & $<120$ & $<180$ & $<250$ & $<80$ \\
\hline $\mathrm{SFR}_{\mathrm{TIR}}\left(M_{\odot} \mathrm{yr}^{-1}\right)$ & $280 \pm 20$ & $315 \pm 25$ & $290 \pm 20$ & $115 \pm 10$ \\
\hline $\operatorname{SFR}_{[\mathrm{C} \mathrm{II}]}\left(M_{\odot} \mathrm{yr}^{-1}\right)$ & $70-440$ & $80-500$ & $85-525$ & $110-700$ \\
\hline
\end{tabular}

Note.

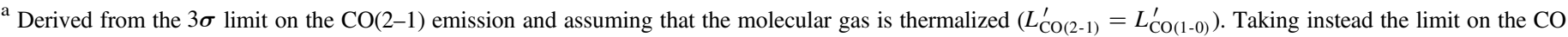

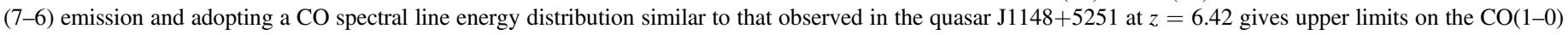
emission that are a factor of 1.0-1.4 smaller.

dynamical mass ratios up to $M_{\mathrm{BH}} / M_{\mathrm{dyn}} \lesssim 25 \%$ and an average of $\left\langle M_{\mathrm{BH}} / M_{\mathrm{dyn}}\right\rangle \approx 2 \%$ (Venemans et al. 2016, and references therein). The high ratios disagree with some simulations of high-redshift quasar host galaxies (e.g., Valiante et al. 2014). Various solutions for this discrepancy have been proposed in the literature. As nearly all high-redshift quasars are selected from flux-limited, optical/near-infrared surveys, by design only the most massive BHs with the highest accretion rates are selected. Also, due to scatter in the correlation between $\mathrm{BH}$ and galaxy mass, the massive BHs of $z \gtrsim 6$ quasars are preferentially found in galaxies that can be an order of magnitude less massive than expected based on the correlation itself (e.g., Willott et al. 2005). Alternatively, FIR emission lines only trace the gas in the inner regions of a galaxy, and using these lines could underestimate the true dynamical mass of the quasar host (e.g., Valiante et al. 2014).

We can also estimate the dynamical mass of the central, unresolved emission. The gas within the central beam with an FWHM of 0 !! $23(1.2 \mathrm{kpc})$ has a velocity dispersion of $\sigma=235 \pm 25 \mathrm{~km} \mathrm{~s}^{-1}$. Setting the radius of this region to $R=0.5 \times \mathrm{FWHM}_{\text {beam }}$, we derive a mass of $M_{\text {dyn,center }}=$ $(1.2 \pm 0.2) \times 10^{10} M_{\odot}$, which is only $\sim 5$ times more than the mass of the $\mathrm{BH}$.

We can compare the dynamical mass with the mass of the molecular gas in the quasar host galaxy. Assuming a gas-todust mass ratio of 100 , the gas mass is $(0.8-4) \times 10^{10} M_{\odot}$, which is $20 \%-95 \%$ of the dynamical mass. In Section 3.4 we showed that a high fraction $(\sim 80 \%)$ of the dust and [C II] emission resides in a compact region with a diameter $<1.5 \mathrm{kpc}$. If this is also the case for the molecular gas, then there is not much room for a massive stellar component in the central $\sim 1-1.5 \mathrm{kpc}$ of the host galaxy, which raises interesting questions regarding the origin of the detected dust emission. Due to the large uncertainties in the molecular gas mass, BH mass, and dynamical mass, we cannot put any strong constraints on the stellar mass in the quasar host galaxy.

\section{Summary}

We present ALMA, PdBI, and VLA observations targeting the dust emission and [C II], [C I], and two CO lines in the host galaxy of quasar $\mathrm{J} 1120+0641$ at $z=7.1$. The ALMA observations of the [C II] line and the underlying continuum greatly increase the spatial resolution compared to earlier measurements with the PdBI (factor of 70 in beam area). Our main findings can be summarized as follows.

1. Within the field of view mapped by ALMA $\left(\sim 25^{\prime \prime}\right)$ the quasar is the only detected source.

2. The dust continuum and [C II] emission regions are very compact and only marginally resolved in the ALMA data. The majority of the emission $(80 \%)$ is associated with a compact region of size $1.2 \times 0.8 \mathrm{kpc}^{2}$ in diameter.

3. The nondetection of the $[\mathrm{CI}]$ line indicates that the heating in the quasar host galaxy is dominated by star formation (PDR), and not by the accreting supermassive $\mathrm{BH}$ (XDR).

4. From the limits on the $\mathrm{CO}(2-1)$ and $\mathrm{CO}(7-6)$ lines we derived upper limits on the molecular gas mass of $M_{\text {gas }}<4 \times 10^{10} M_{\odot}$. This is consistent with a gas-todust mass ratio of $\sim 100$ that is measured in the local universe.

5. We estimate the SFR in the quasar host using both the FIR continuum and the [C II] line measurement. Both methods give consistent results $\left(\mathrm{SFR}_{\mathrm{FIR}} \sim 105-340 M_{\odot} \mathrm{yr}^{-1}\right)$, with a resulting SFR surface density of $\sim 100$ $350 M_{\odot} \mathrm{yr}^{-1} \mathrm{kpc}^{-2}$, well below the value for Eddingtonaccretion-limited star formation (Walter et al. 2009)

6. Surprisingly, the compact [C II] emission does not exhibit ordered motion on kiloparsec scales: applying the virial theorem yields a dynamical mass of the host galaxy of $(4.3 \pm 0.9) \times 10^{10} M_{\odot}$, only $\sim 20 \times$ higher than that of the central supermassive $\mathrm{BH}$. In the very central region, the dynamical mass of the host is only 5 times that of the central $\mathrm{BH}$. In this region, the mass of the $\mathrm{BH}$ and that of the implied dust and gas are able to explain the dynamical 
mass. In other words, there is not much room for a massive stellar component in the very central region. However, we note that the large uncertainties in both the molecular gas and dust mass, as well as the dynamical mass, make it unfeasible to put tight constraints on the stellar mass.

The ALMA observations presented here start to spatially resolve the host galaxy of the most distant quasar known. With the recent commissioning of even longer baselines at ALMA, even higher-resolution imaging of this quasar host is now possible that will start to spatially resolve the sphere of influence of the central supermassive $\mathrm{BH}$.

We thank the referee for providing valuable comments and suggestions. B.P.V. and F.W. acknowledge funding through the ERC grant "Cosmic Dawn." Support for R.D. was provided by the DFG priority program 1573 "The Physics of the Interstellar Medium." P.C.H. and R.G.M. acknowledge the support of the UK Science and Technology Facilities Council (STFC) through the award of a Consolidated Grant to the Institute of Astronomy. We thank Rowin Meijerink for help with the modeling of the line ratios. This paper makes use of the following ALMA data: ADS/ JAO.ALMA\#2012.1.00882.S. ALMA is a partnership of ESO (representing its member states), NSF (USA), and NINS (Japan), together with NRC (Canada) and NSC and ASIAA (Taiwan), in cooperation with the Republic of Chile. The Joint ALMA Observatory is operated by ESO, AUI/NRAO, and NAOJ. Based in part on observations carried out with the IRAM Plateau de Bure Interferometer. IRAM is supported by INSU/CNRS (France), MPG (Germany), and IGN (Spain). The National Radio Astronomy Observatory is a facility of the National Science Foundation operated under cooperative agreement by Associated Universities, Inc.

Facilities: ALMA, IRAM:Interferometer, EVLA.

\section{References}

Aravena, M., Decarli, R., Walter, F., et al. 2016, ApJ, 833, 68 Bañados, E., Venemans, B. P., Decarli, R., et al. 2016, ApJS, 227, 11 Barnett, R., Warren, S. J., Banerji, M., et al. 2015, A\&A, 575, A31 Beelen, A., Cox, P., Benford, D. J., et al. 2006, ApJ, 642, 694 Bertoldi, F., Carilli, C. L., Cox, P., et al. 2003, A\&A, 406, L55 Carilli, C. L., \& Holdaway, M. A. 1999, RaSc, 34, 817 Carilli, C. L., \& Walter, F. 2013, ARA\&A, 51, 105

Carnall, A. C., Shanks, T., Chehade, B., et al. 2015, MNRAS, 451, L16
Contursi, A., Poglitsch, A., Grácia Carpio, J., et al. 2013, A\&A, 549, A118 da Cunha, E., Groves, B., Walter, F., et al. 2013, ApJ, 766, 13

De Looze, I., Cormier, D., Lebouteiller, V., et al. 2014, A\&A, 568, A62 De Rosa, G., Decarli, R., Walter, F., et al. 2011, ApJ, 739, 56

De Rosa, G., Venemans, B. P., Decarli, R., et al. 2014, ApJ, 790, 145

Díaz-Santos, T., Armus, L., Charmandaris, V., et al. 2013, ApJ, 774, 68

Downes, D., \& Solomon, P. M. 1998, ApJ, 507, 615

Draine, B. T., Dale, D. A., Bendo, G., et al. 2007, ApJ, 663, 866

Dunne, L., Eales, S., Edmunds, M., et al. 2000, MNRAS, 315, 115

Fan, X., Strauss, M. A., Becker, R. H., et al. 2006, AJ, 132, 117

Hildebrand, R. H. 1983, QJRAS, 24, 267

Jiang, L., Fan, X., Bian, F., et al. 2009, AJ, 138, 305

Jiang, L., Fan, X., Vestergaard, M., et al. 2007, AJ, 134, 1150

Jiang, L., McGreer, I. D., Fan, X., et al. 2015, AJ, 149, 188

Kaufman, M. J., Wolfire, M. G., Hollenbach, D. J., \& Luhman, M. L. 1999, ApJ, 527, 795

Kormendy, J., \& Ho, L. C. 2013, ARA\&A, 51, 511

Kroupa, P. 2001, MNRAS, 322, 231

Kurk, J. D., Walter, F., Fan, X., et al. 2007, ApJ, 669, 32

Leipski, C., Meisenheimer, K., Walter, F., et al. 2014, ApJ, 785, 154

Maiolino, R., Cox, P., Caselli, P., et al. 2005, A\&A, 440, L51

Matsuoka, Y., Onoue, M., Kashikawa, N., et al. 2016, ApJ, 828, 26

McMullin, J. P., Waters, B., Schiebel, D., Young, W., \& Golap, K. 2007, in ASP Conf. Ser. 376, Astronomical Data Analysis Software and Systems XVI, ed. R. A. Shaw, F. Hill, \& D. J. Bell (San Francisco, CA: ASP), 127

Meijerink, R., \& Spaans, M. 2005, A\&A, 436, 397

Meijerink, R., Spaans, M., \& Israel, F. P. 2007, A\&A, 461, 793

Momjian, E., Carilli, C. L., Walter, F., \& Venemans, B. 2014, AJ, 147, 6

Mortlock, D. J., Patel, M., Warren, S. J., et al. 2009, A\&A, 505, 97

Mortlock, D. J., Warren, S. J., Venemans, B. P., et al. 2011, Natur, 474, 616

Murphy, E. J., Condon, J. J., Schinnerer, E., et al. 2011, ApJ, 737, 67

Priddey, R. S., \& McMahon, R. G. 2001, MNRAS, 324, L17

Reed, S. L., McMahon, R. G., Banerji, M., et al. 2015, MNRAS, 454, 3952

Riechers, D. A., Walter, F., Bertoldi, F., et al. 2009, ApJ, 703, 1338

Rujopakarn, W., Dunlop, J. S., Rieke, G. H., et al. 2016, ApJ, 833, 12 Sandstrom, K. M., Leroy, A. K., Walter, F., et al. 2013, ApJ, 777, 5

Sargsyan, L., Samsonyan, A., Lebouteiller, V., et al. 2014, ApJ, 790, 15

Stefan, I. I., Carilli, C. L., Wagg, J., et al. 2015, MNRAS, 451, 1713

Valiante, R., Schneider, R., Salvadori, S., \& Gallerani, S. 2014, MNRAS, 444, 2442

Venemans, B. P., Bañados, E., Decarli, R., et al. 2015, ApJL, 801, L11

Venemans, B. P., Findlay, J. R., Sutherland, W. J., et al. 2013, ApJ, 779, 24

Venemans, B. P., McMahon, R. G., Walter, F., et al. 2012, ApJL, 751, L25

Venemans, B. P., Walter, F., Zschaechner, L., et al. 2016, ApJ, 816, 37

Walter, F., Bertoldi, F., Carilli, C., et al. 2003, Natur, 424, 406

Walter, F., Riechers, D., Cox, P., et al. 2009, Natur, 457, 699

Walter, F., Weiß, A., Downes, D., Decarli, R., \& Henkel, C. 2011, ApJ, 730, 18

Wang, R., Carilli, C. L., Wagg, J., et al. 2008, ApJ, 687, 848

Wang, R., Wagg, J., Carilli, C. L., et al. 2013, ApJ, 773, 44

Willott, C. J., Albert, L., Arzoumanian, D., et al. 2010a, AJ, 140, 546

Willott, C. J., Bergeron, J., \& Omont, A. 2015, ApJ, 801, 123

Willott, C. J., Delorme, P., Reylé, C., et al. 2010b, AJ, 139, 906

Willott, C. J., Omont, A., \& Bergeron, J. 2013, ApJ, 770, 13

Willott, C. J., Percival, W. J., McLure, R. J., et al. 2005, ApJ, 626, 657

Wu, X.-B., Wang, F., Fan, X., et al. 2015, Natur, 518, 512 